\title{
On Actively Closing Loops in Grid-based FastSLAM
}

\author{
Cyrill Stachniss $^{1} \quad$ Dirk Hähnel ${ }^{1,2} \quad$ Wolfram Burgard $^{1} \quad$ Giorgio Grisetti ${ }^{1,3}$ \\ ${ }^{1}$ University of Freiburg, Department of Computer Science, D-79110 Freiburg, Germany \\ ${ }^{2}$ Intel Research Seattle, 1100 NE 45th Street, Seattle, WA 98105, USA \\ ${ }^{3}$ Dipartimento Informatica e Sistemistica, Universitá "La Sapienza", I-00198 Rome, Italy \\ Correspondence author: Cyrill Stachniss, stachnis@informatik.uni-freiburg.de
}

\begin{abstract}
Acquiring models of the environment belongs to the fundamental tasks of mobile robots. In the past, several researchers have focused on the problem of simultaneous localization and mapping (SLAM). Classical SLAM approaches are passive in the sense that they only process the perceived sensor data and do not influence the motion of the mobile robot. In this paper, we present a novel integrated approach that combines autonomous exploration with simultaneous localization and mapping. Our method uses a grid-based version of the FastSLAM algorithm and considers at each point in time actions to actively close loops during exploration. By re-entering already visited areas, the robot reduces its localization error and in this way learns more accurate maps. Experimental results presented in this paper illustrate the advantage of our method over previous approaches that lack the ability to actively close loops.
\end{abstract}

keywords: exploration, active loop-closure, place re-visiting, re-localization, FastSLAM

\section{Introduction}

In general, the task of acquiring models of unknown environments requires the solution of three subtasks, which are mapping, localization, and motion control. Mapping is the problem of integrating the information gathered with the robot's sensors into a given representation. Localization is the problem of estimating the position of the robot. Finally, the motion control problem involves the question of how to steer a vehicle in order to efficiently guide it to a desired location or along a planned trajectory.

The diagram in Figure 1 depicts also the overlapping areas of these three tasks. Simultaneous localization and mapping, also called SLAM, is the problem of building a map based on pose estimates while simultaneously localizing the robot within the map constructed so far. Active localization seeks to guide the robot to locations within the map to improve the pose estimate. In contrast to this, exploration approaches focus on guiding the robot efficiently through the environment in order to build a map. The center area of the diagram represents the so-called integrated approaches which address mapping, localization, and motion control simultaneously. 


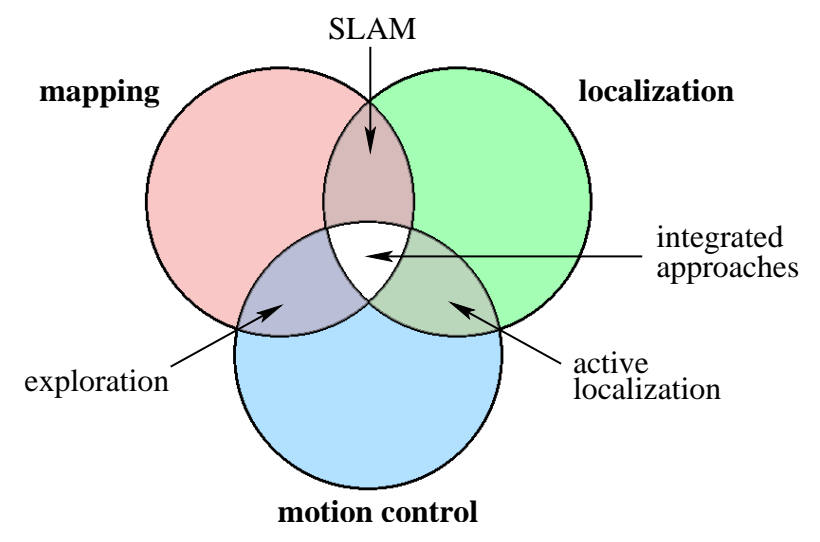

Figure 1: Sub-tasks that need to be solved by a robot in order to acquire accurate models of the environment [14]. The overlapping areas represent combinations of these sub-tasks.
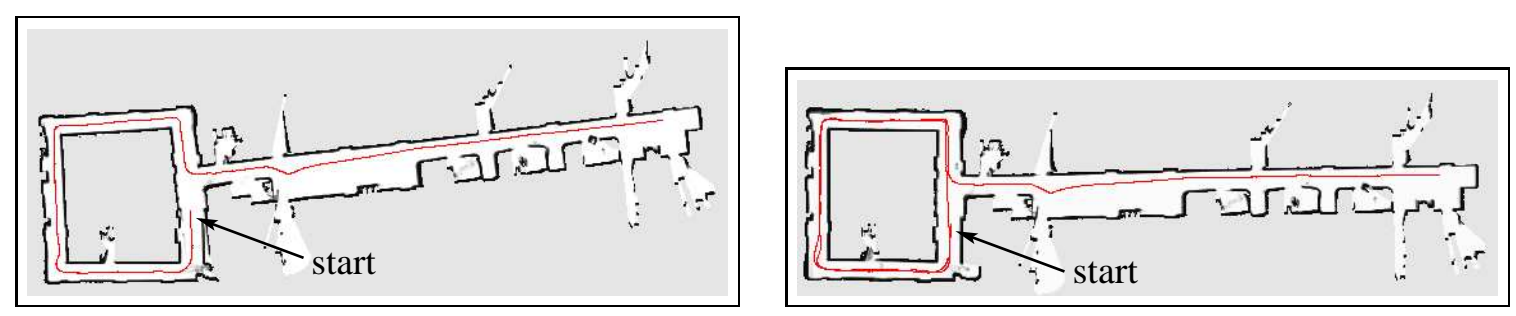

Figure 2: This figure shows two maps obtained from real world data acquired at Sieg Hall, University of Washington. The left image depicts an experiment in which the robot traversed the loop only once before it entered the long corridor. As can be seen, the robot was unable to correctly close the loop, which leaded to an error of 7 degrees in the orientation of the horizontal corridor. In the case in which the robot re-visited the loop, the orientation error was reduced to 1 degree (see right image).

A naive approach to realize an integrated technique which solves all three tasks simultaneously, could be to combine a SLAM algorithm with an exploration procedure. Since exploration strategies often try to cover unknown terrain as fast as possible, they avoid repeated visits to known areas. This strategy, however, is suboptimal in the context of the SLAM problem because the robot typically needs to re-visit places to localize itself again. A good pose estimate is necessary to make the correct data association, i.e., to determine if the current measurements fit into the map built so far. If the robot uses an exploration strategy that avoids multiple visits to the same place, the probability of making the correct associations is reduced. This indicates that combinations of exploration strategies and SLAM algorithms should consider whether it is worth re-entering already covered spaces or to explore new terrain. It can be expected that a system, which takes this decision into account, can improve the quality of the resulting map.

Figure 2 gives an example that illustrates why an integrated approach performing active place revisiting provides better results than approaches that do not consider re-entering known terrain during the exploration phase. In the situation shown in the left image, the robot traversed the loop just once. 
The robot was not able to correctly determine the angle between the loop and the straight corridor because it did not collect enough data to accurately localize itself. The second map shown in the right image has been obtained with the approach described in this paper after the robot traveled twice around the loop before entering the corridor. As can be seen from the figure, this reduces the orientation error from approximately 7 degrees (left image) to 1 degree (right image). This example illustrates that the capability to actively close loops during exploration allows the robot to reduce its pose uncertainty during exploration and thus to learn more accurate maps.

The contribution of this paper is an integrated algorithm for generating trajectories to actively close loops during SLAM and exploration. Our algorithm uses a grid-based version of the FastSLAM algorithm and maintains a Rao-Blackwellized particle filter to estimate the map and the trajectory of the robot. Our approach explicitely takes into account the uncertainty about the pose of the robot during the exploration task. Additionally, it reduces the risk that the robot becomes overly confident in its pose when actively closing loops, which is a typical problem of particle filters in this context. As a result, we obtain more accurate maps compared to combinations of SLAM with greedy exploration.

This paper is organized as follows. The next section discusses related work and in Section 3 we then explain the idea of grid-based FastSLAM, the mapping algorithm used throughout this work. In Section 4, we present our integrated exploration technique. We describe how to take into account the pose uncertainty and how to actively close loops. Finally, Section 5 presents experiments carried out on real robots as well as in simulation.

\section{Related Work}

Several previous approaches to SLAM and mobile robot exploration are related to our work. In the context of exploration, most of the techniques presented so far focus on generating motion commands that minimize the time needed to cover the whole terrain $[2,12,23,24]$. Other methods seek to optimize the view-points of the robot to maximize the expected information gain and to minimize the uncertainty of the robot about grid cells [8,20]. Most of these techniques, however, assume that the location of the robot is known during exploration. In the area of SLAM, the vast majority of papers focuses on the aspect of state estimation as well as belief representation and update $[4,5,6,10,11,15,17,21]$. These techniques, however, are passive and only consume incoming sensor data without explicitely generating controls.

Recently, some techniques have been proposed which actively control the robot during SLAM. For example, Makarenko et al. [14] as well as Bourgault et al. [1] extract landmarks out of laser range scans and use an Extended Kalman Filter to solve the SLAM problem. Furthermore, they introduce a utility function which trades off the cost of exploring new terrain with the utility of selected positions with respect to a potential reduction of uncertainty. The approaches are similar to the work done by Feder et al. [7] who consider local decisions to improve the pose estimate during mapping. Sim et al. [19] presented an approach in which the robot follows a parametric curve to explore the environment 
and considers place re-visiting actions if the pose uncertainty gets too high. These four techniques integrate the uncertainty in the pose estimate of the robot into the decision process of where to move next. However, they rely on the fact that the environment contains landmarks that can be uniquely determined during mapping.

In contrast to this, the approach presented in this paper makes no assumptions about distinguishable landmarks in the environment. It uses raw laser range scans to compute accurate grid maps. It considers the utility of re-entering known parts of the environment and following an encountered loop to reduce the uncertainty of the robot in its pose. In this way, the resulting maps become highly accurate.

\section{Grid-Based FastSLAM}

To estimate the map of the environment, we use a highly efficient variant of the FastSLAM algorithm [15], which itself is an extension of the Rao-Blackwellized particle filter for simultaneous localization and mapping proposed by Murphy et al. [5]. FastSLAM uses a set of weighted particles to represent the full posterior $p\left(x_{1: t}, m \mid z_{1: t}, u_{0: t-1}\right)$ about the map $m$ of the environment and the trajectory $x_{1: t}$ of the robot given a sequence of observations $z_{1: t}$ up to time $t$ and the odometry measurements $u_{0: t-1}$. The key idea of the Rao-Blackwellized particle filter for SLAM is to separate the estimation of the trajectory of the robot from the map estimation process

$$
\begin{aligned}
p\left(x_{1: t}, m \mid z_{1: t}, u_{0: t-1}\right) & =p\left(x_{1: t} \mid z_{1: t}, u_{0: t-1}\right) p\left(m \mid x_{1: t}, z_{1: t}, u_{0: t-1}\right) \\
& =p\left(x_{1: t} \mid z_{1: t}, u_{0: t-1}\right) p\left(m \mid x_{1: t}, z_{1: t}\right)
\end{aligned}
$$

where Eq. (2) is obtained from Eq. (1) by assuming that $m$ is independent of the odometry measurements $u_{0: t-1}$ given all the poses $x_{1: t}$ of the robot and the corresponding observations $z_{1: t}$.

To estimate the first term of Eq. (2), namely the posterior $p\left(x_{1: t} \mid z_{1: t}, u_{0: t-1}\right)$, FastSLAM uses a particle filter. This filter estimates the trajectory of the robot based on the odometry information and the observed laser range data similar to Monte-Carlo localization [3].

Estimating the full posterior about the map and poses of the robot can be done efficiently since the quantity $p\left(m \mid x_{1: t}, z_{1: t}\right)$ can be computed analytically once $x_{1: t}$ and $z_{1: t}$ are known. By assuming that laser range finders provide accurate range observations and given one knows the poses of the robot while obtaining these observations, a grid map can be directly computed using ray-casting operations. As a result, each of the samples represents a possible trajectory of the robot and additionally maintains an individual map which is updated upon "mapping with known poses" [16] according to its own pose estimate.

One of the most common particle filtering algorithms is the Sampling Importance Resampling (SIR) filter. FastSLAM incrementally processes the observations and the odometry readings as they are available and updates the set of samples representing the posterior about the map and the trajectory of the vehicle. The overall process can be summarized by the following four steps: 


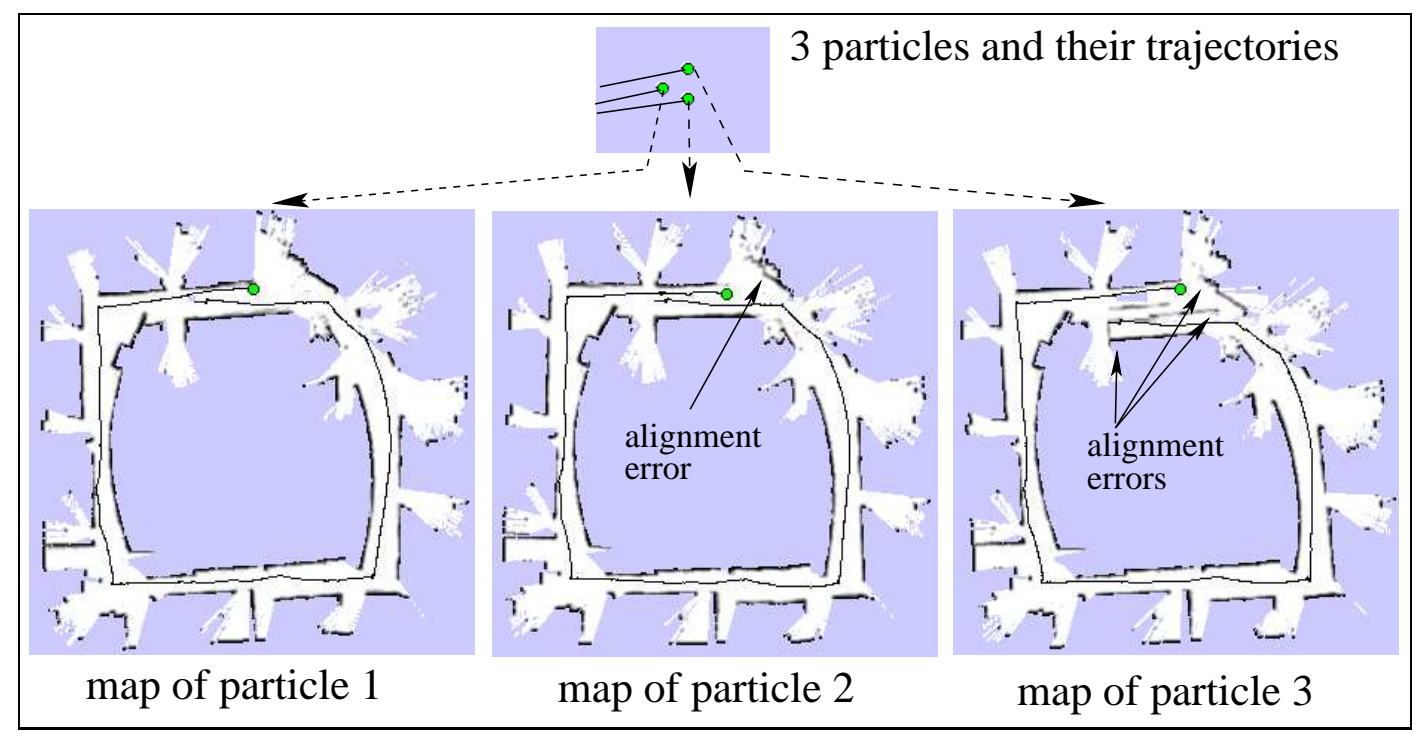

Figure 3: Example for three particles used within FastSLAM to represent $p\left(x_{1: t}, m \mid z_{1: t}, u_{0: t-1}\right)$. Each particle estimates the trajectory of the robot and maintains an individual map which is updated according to the estimated trajectory.

1. Sampling: The next generation of particles is obtained from the current generation, by sampling from a proposal distribution $\pi$. The motion model of the robot is often used as the proposal.

2. Importance Weighting: An individual importance weight $\omega$ is assigned to each particle. The weights account for the fact that the proposal distribution $\pi$ in general is not equal to the true distribution of successor states. In our filter, the weight of each particle is proportional to the likelihood $p\left(z_{t} \mid m, x_{t}\right)$ of the most recent observation given the map $m$ associated with this particle and the corresponding pose $x_{t}$.

3. Resampling: Particles with a low importance weight $\omega$ are typically replaced by samples with a high weight. This step is necessary since only a finite number of particles are used to approximate a continuous distribution. Furthermore, resampling allows to apply a particle filter in situations in which the true distribution differs from the proposal.

4. Map Estimating: For each particle, the corresponding map estimate is updated based on the obtained observation and the pose represented by that particle.

The FastSLAM algorithm used throughout this paper computes grid maps. It applies a scanmatching procedure to compute highly accurate odometry data and uses this corrected odometry in the prediction step of the particle filter [11]. In this way, the number of particles can be reduced so that even maps of large environments can be constructed online. An example for such a filter is illustrated in Figure 3. It depicts three particles with the individually estimated trajectories and the maps updated according to the estimated trajectory. In the depicted situation, the robot closed a loop and the different particles produced different maps. Particle 1 generated an quite accurate pose estimate, whereas 
particles 3 yields big alignments errors. Therefore, particle 1 will get a much higher importance weight compared to particle 3 . The weight of particle 2 will by between the weight of particle 1 and 3 because its alignment error is smaller than the one of particle 3 but bigger than the one of particle 1.

Note that adapting the map discretization leads to only minor changes in the resulting trajectory. Generally, the finer the resolution, the more precise is typically the pose estimate and therefore the resulting map. However, our observation is that as long as the grid resolution is reasonable small, it mainly influences the execution time of the algorithm and the memory requirements. Typically, the grid resolution is set values between $5 \mathrm{~cm}$ and $15 \mathrm{~cm}$.

In the following section, we describe how to actively close loops during exploration in order to obtain accurate grid maps using the FastSLAM algorithm.

\section{Exploration With Active Loop-Closing for FastSLAM}

During FastSLAM, whenever the robot explores new terrain, all samples have more or less the same importance weight, since the most recent measurement is typically consistent with the part of the map constructed from the immediately preceding observations. As a result, the uncertainty of the particle filter increases. As soon as it re-enters known terrain, however, the maps of some particles are consistent with the current measurement and some are not. Accordingly the weights of the samples differ largely. Due to the resampling step, the uncertainty about the pose of the robot usually decreases. One typical example is shown in Figure 4. In the two left images, the robot explores new terrain and the uncertainty of the sample set increases. In the right image, the robot travels through known terrain and unlikely particles have vanished.

Note that this effect is much smaller if the robot just moves backward a few meters to re-visit previously scanned areas. This is because each map associated with a particle is generally locally consistent. Inconsistencies mostly arise when the robot re-enters areas explored some time ago. Therefore, visiting places seen further back in the history has a stronger effect on the differences between the importance weights and typically also on the reduction of uncertainty compared to places recently observed.

\subsection{Detecting Opportunities to Close Loops}

The key idea of our approach is to identify opportunities for closing loops during terrain acquisition. Here, closing a loop means actively re-entering the known terrain and following a previously traversed path. To determine whether there exists a possibility to close a loop we consider two different representations of the environment. In our current system, we associate with particle $s$ an occupancy grid map $m^{[s]}$ and a topological map $\mathcal{G}^{[s]}$, both of which are updated while the robot is performing the exploration task. In the topological map $\mathcal{G}^{[s]}$, the vertices represent positions visited by the robot. The edges represent the trajectory corresponding to the particle $s$. To construct the topological map, we initialize it with one node corresponding to the starting location of the robot. Let $x_{t}^{[s]}$ be the pose of 

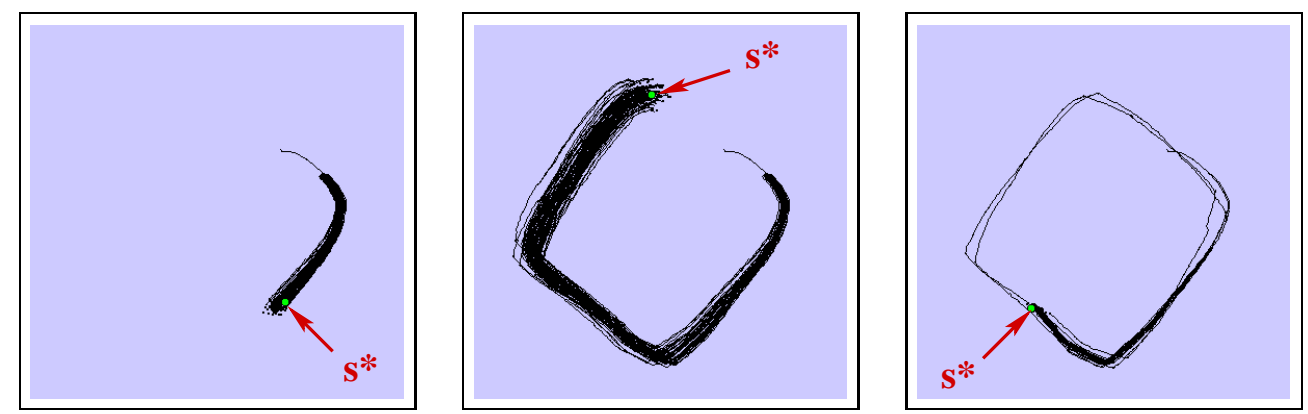

Figure 4: Evolution of a particle set and the map of the particle $s^{*}$ at three different time steps. In the two left images, the vehicle traveled through unknown terrain, so that the uncertainty increased. In the right image, the robot re-entered known terrain so that samples representing unlikely trajectories vanished.

particle $s$ at the current time step $t$. We add a new node at $x_{t}^{[s]}$ to $\mathcal{G}^{[s]}$ if the distance between $x_{t}^{[s]}$ and all other nodes in $\mathcal{G}^{[s]}$ exceeds a threshold $c$ (here set to $2.5 \mathrm{~m}$ ) or if none of the other nodes in $\mathcal{G}^{[s]}$ is visible from $x_{t}^{[s]}$ :

$$
\forall n \in \operatorname{nodes}\left(\mathcal{G}^{[s]}\right) \quad: \quad\left[\operatorname{dist}_{m[s]}\left(x_{t}^{[s]}, n\right)>c \quad \vee \quad \operatorname{not}_{-} \operatorname{visible}_{m^{[s]}}\left(x_{t}^{[s]}, n\right)\right] .
$$

Whenever a new node is created, we also add an edge from this node to the most recently visited node. To determine whether or not a node is visible from another node, we perform a ray-casting operation in the occupancy grid $m^{[s]}$.

Figure 5 depicts such a graph for one particular particle during different phases of an exploration task. In each image, the topological map $\mathcal{G}^{[s]}$ is printed on top of metric map $m^{[s]}$. To motivate the idea of our approach, we would like to refer the reader to the left image of this figure. Here, the robot was almost closing a loop. This can be detected by the fact that the length of the shortest path between the current pose of the robot and previously visited locations in the topological map $\mathcal{G}^{[s]}$ was large, whereas it was small in the grid-map $m^{[s]}$.

Thus, to determine whether or not a loop can be closed, we compute for each sample $s$ the set $\mathcal{I}(s)$ of positions of interest which contains all nodes that are close to the current pose $x_{t}^{[s]}$ of particle $s$ based on the grid map $m^{[s]}$, but are far away given the topological map $\mathcal{G}^{[s]}$ of $s$

$$
\mathcal{I}(s)=\left\{x_{t^{\prime}}^{[s]} \in \operatorname{nodes}\left(\mathcal{G}^{[s]}\right) \mid \operatorname{dist}_{m^{[s]}}\left(x_{t^{\prime}}^{[s]}, x_{t}^{[s]}\right)<c_{1} \wedge \operatorname{dist}_{\mathcal{G}^{[s]}}\left(x_{t^{\prime}}^{[s]}, x_{t}^{[s]}\right)>c_{2}\right\}
$$

Here, $\operatorname{dist}_{\mathcal{M}}\left(x_{1}, x_{2}\right)$ is the length of the shortest path from $x_{1}$ to $x_{2}$ given the representation $\mathcal{M}$. The distance between two nodes in $\mathcal{G}^{[s]}$ is given by the length of the shortest path between both nodes, whereas the length of a path is computed by the sum over the lengths of the traversed edges. Depending on the number of nodes in $\mathcal{I}(s)$, this distance information can be efficiently computed using either the $A^{*}$ algorithm or Dijkstra's algorithm. The terms $c_{1}$ and $c_{2}$ are constants that must satisfy the constraint $c_{1}<c_{2}$. In our current implementation, the values of these constants are $c_{1}=6 \mathrm{~m}$ and $c_{2}=20 \mathrm{~m}$. 

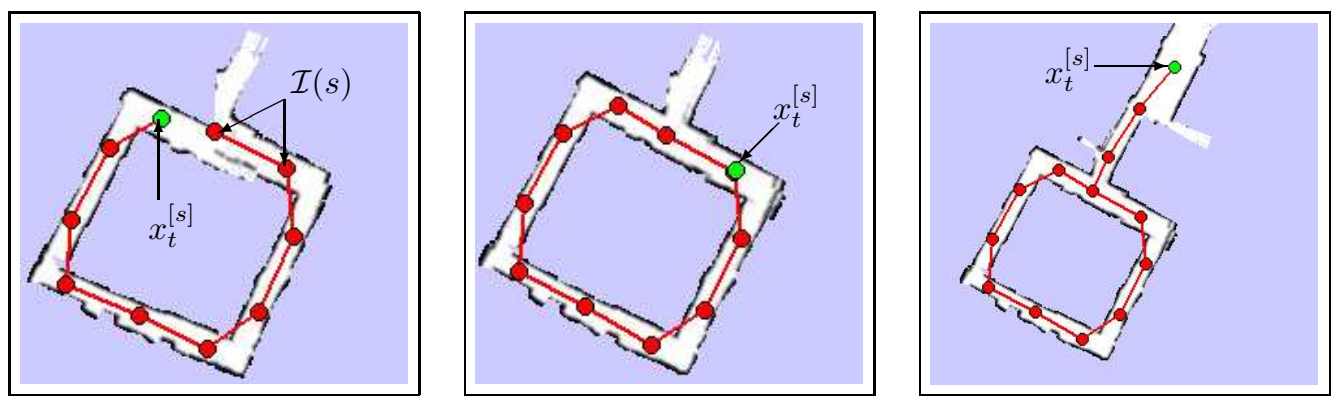

Figure 5: The red dots and lines in these three image represent the nodes and edges of $\mathcal{G}^{[s]}$. In the left image, $\mathcal{I}(s)$ contained two nodes (indicated by the arrows) and in the middle image the robot closed the loop until the pose uncertainty is reduced. After this, it continued with the acquisition of unknown terrain (right image).

If $\mathcal{I}(s) \neq \emptyset$, there exist so-called shortcuts from the current pose $x_{t}^{[s]}$ represented by the corresponding particle to the positions in $\mathcal{I}(s)$. These shortcuts represent edges that would close a loop in the topological map $\mathcal{G}^{[s]}$. The left image of Figure 5 illustrates a situation in which a robot encounters the opportunity to close a loop since $\mathcal{I}(s)$ contains two nodes which is indicated by two arrows. The key idea of our approach is to use such shortcuts whenever the uncertainty of the robot in its pose becomes too large. The robot then re-visits portions of the previously explored area and in this way reduces the uncertainty in its position.

To determine the most likely movement allowing the robot to follow a previous path of a loop, one in principle has to integrate over all particles and consider all potential outcomes of that particular action. Since this would be too time consuming for online-processing, we consider only the particle $s^{*}$ with the highest accumulated logarithmic observation likelihood:

$$
s^{*}=\underset{s}{\operatorname{argmax}} \sum_{i=1}^{t} \log p\left(z_{i} \operatorname{midm}^{[s]}, x_{i}^{[s]}\right) .
$$

If $\mathcal{I}\left(s^{*}\right) \neq \emptyset$, we choose the node $x_{t_{e}}$ from $\mathcal{I}\left(s^{*}\right)$ which is closest to $x_{t}^{\left[s^{*}\right]}$

$$
x_{t_{e}}=\underset{x \in \mathcal{I}\left(s^{*}\right)}{\operatorname{argmin}} \operatorname{dist}_{m^{\left[s^{*}\right]}}\left(x_{t}^{\left[s^{*}\right]}, x\right)
$$

In the sequel, $x_{t_{e}}$ is denoted as the entry point at which the robot has the possibility to close a loop. $t_{e}$ corresponds to the last time the robot was at the node $x_{t_{e}}$.

Note that it can happen that the particle $s^{*}$ is far away from representing the correct pose estimate. In such a situation, the robot sometimes computes a path, which cannot be traversed in practice. The robot will follow this path until it recognizes that it is blocked and then will abort the loop closing behavior. 

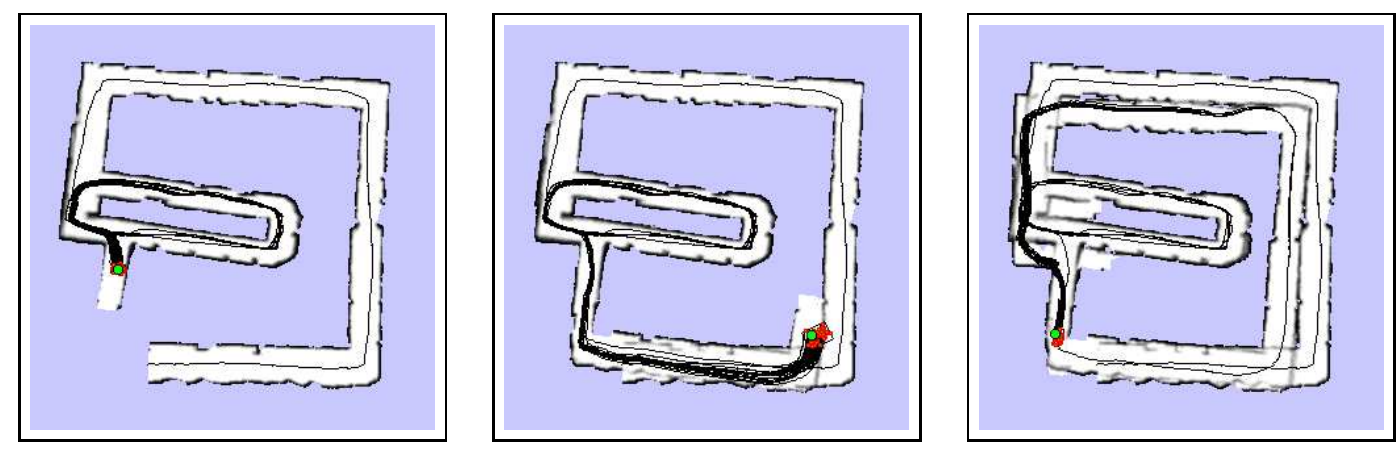

Figure 6: The particle depletion problem: A robot traveled through the inner loop several times (left image). After this, the diversity of hypotheses about the trajectory outside the inner loop had decreased too much (middle image) and the robot is unable to close the outer loop correctly (right image).

\subsection{Stopping the Loop-Closing Process}

To determine whether or not the robot should activate the loop-closing behavior, our system constantly monitors the uncertainty $\mathcal{H}(t)$ about the robot's pose at the current time step. The necessary condition for starting the loop-closing process is the existence of an entry point $x_{t_{e}}$ and that $\mathcal{H}(t)$ exceeds a given threshold. Once the loop-closing process has been activated, the robot approaches $x_{t_{e}}$ and then follows the path taken after previously arriving at $x_{t_{e}}$. During this process, the uncertainty in the pose of the vehicle typically decreases because the robot is able to localize itself in the map built so far and unlikely particles vanish.

Furthermore, we have to define a criterion for deciding when the robot actually has to stop following a loop. A first attempt could be to introduce a threshold and to simply stop the trajectory following behavior as soon as the uncertainty becomes smaller than a given threshold. This criterion, however, can be problematic especially in the case of nested loops. Suppose the robot encounters the opportunity to close a loop that is nested within an outer and so far unclosed loop. If it eliminates all of its uncertainty by repeatedly traversing the inner loop, particles necessary to close the outer loop may vanish. As a result, the filter diverges and the robot fails to build a correct map (compare Figure 6).

To remedy this so-called particle depletion problem [22], we introduce a constraint on the uncertainty of the robot. Let $\mathcal{H}\left(t_{e}\right)$ denote the uncertainty of the posterior when the robot visited the entry point last time. Then the new constraint allows the robot to re-traverse the loop only as long as its current uncertainty $\mathcal{H}(t)$ exceeds $\mathcal{H}\left(t_{e}\right)$. If the constraint is violated the robot resumes its terrain acquisition (exploration) process. This constraint is designed to reduce the risk of depleting relevant particles during the loop-closing process. The idea is that by observing the area within the loop, the robot does not obtain any information about the world outside the loop. As a result, the robot cannot reduce the uncertainty $\mathcal{H}(t)$ in its current posterior below its uncertainty $\mathcal{H}\left(t_{e}\right)$ when entering the loop since $\mathcal{H}\left(t_{e}\right)$ is the uncertainty stemming from the world outside the loop.

To better illustrate the importance of this constraint, consider the following example: A robot moves 
from place $A$ to place $B$ and then repeatedly observes $B$. While it is mapping $B$, it does not get any further information about $A$. Since each particle represents a whole trajectory of the robot, hypotheses representing ambiguities about $A$ will also vanish when reducing potential uncertainties about $B$. Our constraint reduces the risk of depleting particles representing ambiguities about $A$ by aborting the loop-closing behavior at $B$ as soon as the uncertainty drops below the uncertainty stemming from $A$.

Finally, we have to describe how we actually measure the uncertainty in the position estimate. The typical way of measuring the uncertainty of a posterior is to use the entropy. To compute the entropy of a posterior represented by particles, one typically uses a multi-dimensional grid representing the possible (discretized) states. Each cell $a$ in this grid stores a probability which is given by the sum of the normalized weights of the samples corresponding to that cell. The entropy is then computed by summing up $-p(a) \log p(a)$ of each cell $a$ in that grid.

In the case of multi-modal distributions, however, the entropy does not consider the distance between the different modes. As a result, a set of $k$ different pose hypotheses which are located close by each other leads to the same entropy value than the situation in which $k$ hypotheses are randomly distributed over the environment. The resulting maps, however, would look similar in the first case, but quite different in the second case. In our experiments, we figured out that we obtain better results if we use the volume expanded by the samples instead of the entropy of the posterior. We therefore calculate the pose uncertainty by determining the volume of the oriented bounding box around the particle cloud. A good approximation of the minimal oriented bounding box can be obtained efficiently by a principal component analysis.

Note that the loop-closing process is also aborted after a robot traveled for a long period of time in the same loop in order to avoid a - theoretically possible - endless loop-closing behavior. In all our experiments, however, this problem has never been encountered.

\subsection{Reducing the Exploration Time}

The experiments presented later on in this paper demonstrate that our uncertainty based stopping criterion is an effective way to reduce the risk of particle depletion. However, it can happen that newly acquired sensor data acquired during loop-closing does not provide a lot of new information for the robot. Moving through such terrain leads to an increased exploration time. Therefore, it would be more efficient to abort the loop-closing in situations in which the new sensor data does not help to identify unlikely hypotheses.

To estimate how well the current set of $N$ particle represents the true posterior, Liu [13] introduced the effective number of particles $N_{\text {eff }}$ (also called effective sample size):

$$
N_{\text {eff }}(t)=\frac{1}{\sum_{s=1}^{N}\left(\omega_{t}^{[s]}\right)^{2}}
$$

The idea behind this measure is to determine the variance in the importance weights of the particles. Liu uses $N_{\text {eff }}$ to resample in an intelligent way but it is also useful in the context of active loop- 
closing. We monitor the change of $N_{\text {eff }}$ over time, which allows us to analyze how the new acquired information affects the filter. If $N_{\text {eff }}$ stays constant, the new information does not help to identify unlikely hypotheses represented by the individual particles. In that case, the variance in the importance weights of the particles does not change over time. If, in contrast, the value of $N_{\text {eff }}$ decreases over time, the new information is used to identify that some particles are less likely than others. This is exactly the criterion we need to decide whether or not the loop-closing should be aborted in order to keep the exploration time small. As long as new information helps to identify unlikely particles, we follow the loop. As soon as the observations do not provide any new knowledge about the environment for a period of $k$ time steps, we continue to explore new terrain.

Note that this criterion is optional and in not essential for a successful loop-closing strategy. It can directly be used if the underlying FastSLAM approach applies an adaptive resampling technique ${ }^{1}$. More details on Rao-Blackwellized mapping using adaptive resampling have been published by Grisetti et al. [9]. In the experimental section of this paper, we will demonstrate that $N_{\text {eff }}$ is a useful criterion in the context of active loop-closing and how it behaves during exploration.

As long as the robot is localized well enough or no loop can be closed, we use a frontier-based exploration strategy [2] to choose target points for the robot. A frontier is any known and unoccupied cell that is an immediate neighbor of an unknown, unexplored cell [24]. By extracting frontiers from a given grid map, one can easily determine potential target locations which lead the robot to so far unknown terrain. To select one of these frontier cells as the next goal location, a common way is to determine the travel cost to each frontier cell. This cost can be computed using Dijkstra's algorithm or a value iteration technique like done in [2]. It has been shown by Koenig and Tovey [12], that for a single robot this strategy yields reasonable short trajectories compared to the theoretically optimal solution. In our current system, we determine frontiers based on the map of the particle $s^{*}$.

A precise formulation of the loop-closing strategy is given by Algorithm 1. In our implementation, this algorithm runs as a background process that is able interrupt the frontier-based exploration procedure.

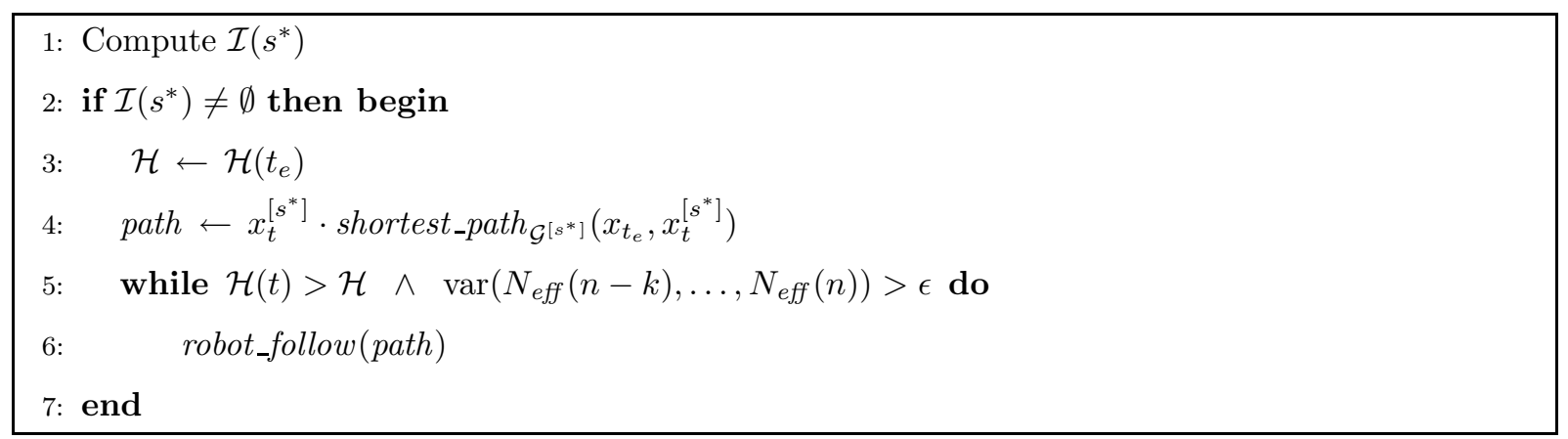

Algorithm 1: The loop-closing algorithm

\footnotetext{
${ }^{1}$ If no adaptive resampling is used, one needs to monitor the relative change in $N_{\text {eff }}$ after integrating each measurements, because after each resampling step the weights of all particles are set to $\frac{1}{N}$. As a result, $N_{\text {eff }}$ is always equal to the number $N$ of particles and the variance therefore is zero.
} 

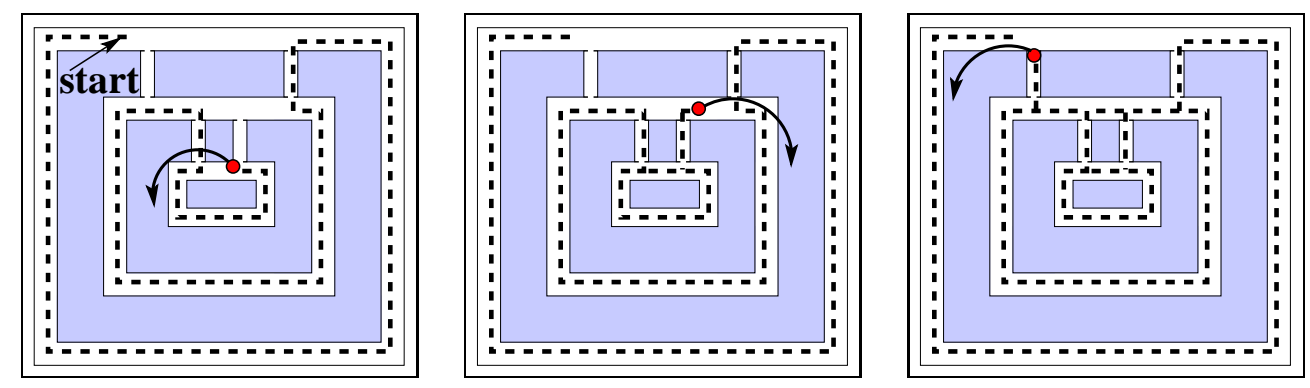

Figure 7: Active loop-closing of multiple nested loops.

\subsection{Handling Multiple Nested Loops}

Note that our loop-closing technique can also handle multiple nested loops. During the loop-closing process, the robot follows its previously taken trajectory to re-localize. It does not leave this trajectory until the termination criterion (see line 5 in Algorithm 1) is fulfilled. Therefore, it never starts a new loop-closing process before the current one is completed. A typical example with multiple nested loops is illustrated in Figure 7. In the situation depicted in the left picture, the robot starts with the loop-closing process for the inner loop. After completing the most inner loop, it moves to the second inner one and again starts the loop-closing process. Since our algorithm considers the uncertainty at the entry point, it keeps enough variance in the filter to also close the outer loop correctly. In general, the quality of the solution and whether or not the overall process succeeds depends on the number of particles used. Since determining this quantity is an open research problem, the number of particles has to be defined by the user in our current system.

\section{$5 \quad$ Experiments}

Our approach has been implemented and evaluated in a series of real world and simulation experiments. For the real world experiments we used an iRobot B21r robot and an ActivMedia Pioneer II robot. Both are equipped with a SICK laser range finder. For the simulation experiments we used the realtime simulator of the Carnegie Mellon Robot Navigation Toolkit [18].

The experiments described in this section are designed to illustrate that our approach can be used to actively learn accurate maps of large indoor environments. Furthermore, they demonstrate that our integrated approach yields better results than an approach without active loop-closing. Additionally, we analyze how the active termination of the loop-closure influences the result of the mapping process.

\subsection{Real World Exploration}

The first experiment was carried out to illustrate that our current system can effectively control a mobile robot to actively close loops during exploration. To perform this experiment, we used a Pioneer II robot to explore the main lobby of the Department for Computer Science at the University of Freiburg. The 

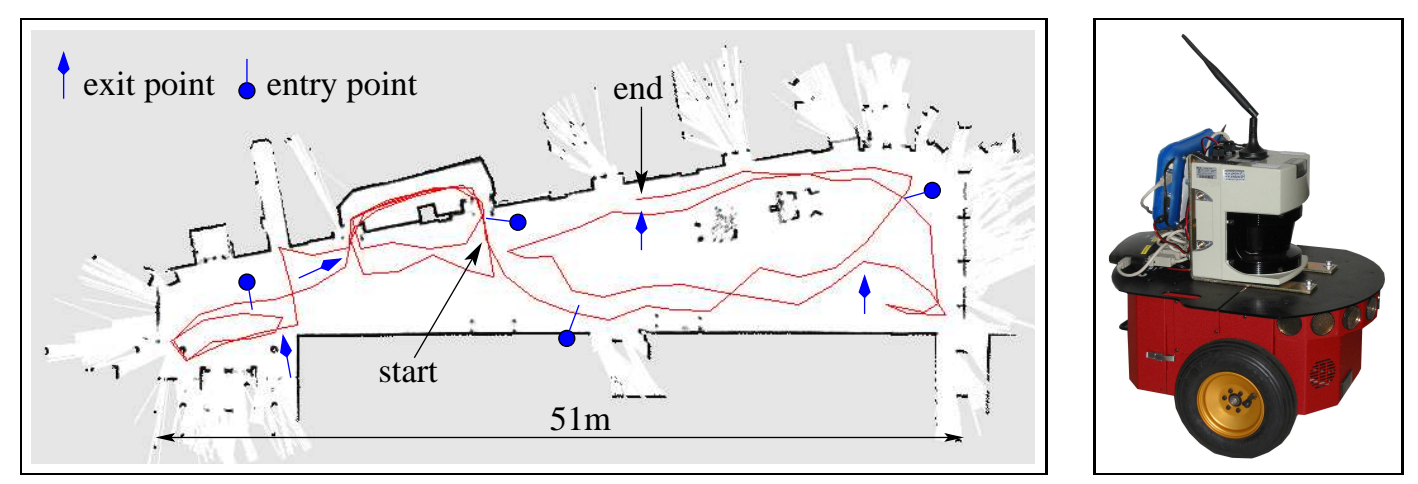

Figure 8: The left image shows the resulting map of an exploration experiment in the entrance hall of the Department for Computer Science at the University of Freiburg. It was carried out using a Pioneer II robot equipped with a laser range scanner (see right image). Also plotted is the path of the robot as well as entry and exit points where the robot started and stopped the active loop-closing process.
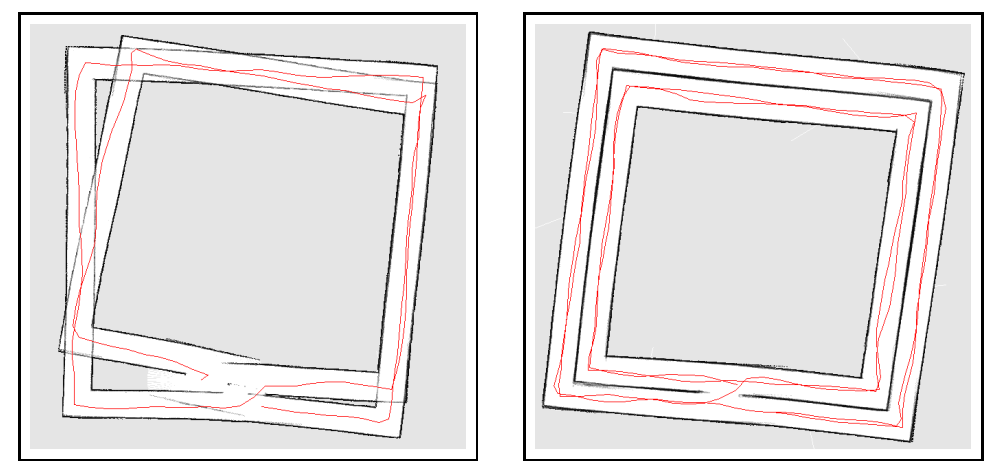

Figure 9: This figure depicts an environment with two large loops. The outer loop has a length of over $220 \mathrm{~m}$. The left image show the resulting map of a trajectory in which the robot drove through the loops only once. In the second run, the robot visited every loop twice and obtained a highly accurate map (see right image).

size of this environment is $51 \mathrm{~m}$ by $18 \mathrm{~m}$. Figure 8 depicts the final result obtained by a completely autonomous exploration run using our active loop-closing technique. It also depicts the trajectory of the robot, which has an overall length of $280 \mathrm{~m}$. The robot decided four times to re-enter a previously visited loop in order to reduce the uncertainty in its pose. Figure 8 also shows the corresponding entry points as well as the positions where the robot left the loops ("exit points"). In this experiment, the FastSLAM routine used 250 particles. As can be seen, the resulting map is quite accurate.

\subsection{Active Loop-Closing vs. Frontier-Based Exploration}

The second experiment should illustrate the difference in approaches that do not consider loop-closing actions. We used real robot data obtained with a B21r robot in the Sieg Hall at the University of Washington. As can be seen from the motivating example in the introduction (Figure 2), the robot 
traversed the loop twice during map building. To eliminate the influence of measurement noise and different movements of the robot, we removed the data corresponding to one loop traversal from the recorded data file and used this data as input to our FastSLAM algorithm. In this way, we simulated the behavior of a greedy exploration strategy which forces the robot to directly enter the corridor after returning to the starting location in the loop. As can be seen from the same figure, an approach that does not actively re-enter the loop fails to correctly estimate the angle between the loop and the corridor which should be oriented horizontally in that figure. Whereas the angular error was 7 degrees with the standard approach, it was only 1 degree in the case where the robot traversed the loop twice. Both maps corresponded to the particle with the highest accumulated importance factor.

A further experiment that illustrates the advantage of place re-visiting is shown in Figure 9. The environment used in this simulation run is $80 \mathrm{~m}$ by $80 \mathrm{~m}$ and contains two large nested loops with nearly featureless corridors. The left image shows the result of the frontier-based approach which traversed each loop only once. Since the robot is not able to correct the accumulated pose error, the resulting map contains large inconsistencies and two of the corridors are mapped onto each other. Our approach, in contrast, first revisits the outer loop before entering the inner one (see right image). Accordingly, the resulting map is quite accurate.

\subsection{A Quantitative Analysis}

To quantitatively evaluate the advantage of the loop-closing behavior, we performed a series of simulation experiments in an environment similar to Sieg Hall. We performed 20 experiments, 10 with active loopclosing and 10 without. After completing the exploration task, we measured the average error in the relative distances between positions lying on the resulting estimated trajectory and the ground truth provided by the simulator. The results are depicted in Figure 10. As can be seen, the active loop-closing behavior significantly reduces the error in the position of the robot.

\subsection{Importance of the Termination Criterion}

In this experiment, we analyze the importance of the constraint that terminates the active loop-closing behavior as soon as the current uncertainty $\mathcal{H}(t)$ of the belief drops under the uncertainty $\mathcal{H}\left(t_{e}\right)$ of the posterior when the robot was at the entry point the last time.

In this simulated experiment, the robot had to explore an environment which contains two nested loops and is depicted in Figure 11 (d). In the first case, we simply used a constant threshold to determine whether or not the loop-closing behavior should be stopped. In the second case, we applied the additional constraint that the uncertainty should not become smaller than $\mathcal{H}\left(t_{e}\right)$.

Figure 6 shows the map of the particle with the highest accumulated importance weight obtained with our algorithm using a constant threshold instead of considering $\mathcal{H}\left(t_{e}\right)$. In this case, the robot repeatedly traversed the inner loop (left image) until its uncertainty was reduced below a certain threshold. After three and a half rounds it decided to again explore unknown terrain, but the diversity of hypotheses 


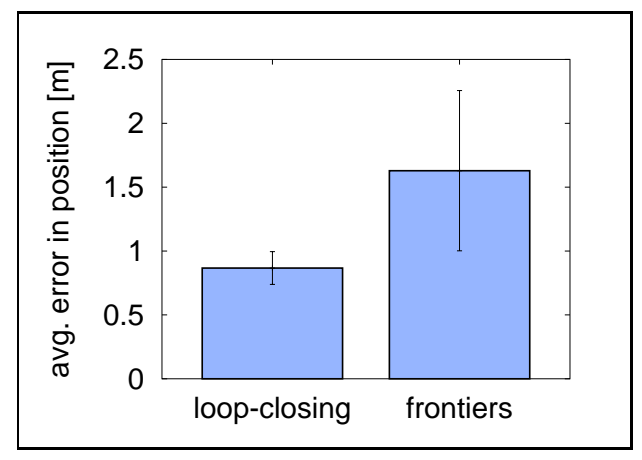

Figure 10: This figure compares our loop-closing strategy with a pure frontier-based exploration technique. The left bar in this graph plots the average error in the pose of the robot obtained with our loop-closing strategy. The right one shows the average error when a frontier-based approach was used. As can be seen, our technique significantly reduces the distances between the estimated positions and the ground truth (confidence intervals do not overlap).

had decreased too much (middle image). Accordingly the robot was unable to accurately close the outer loop (right image). We repeated this experiment several times and in none of the cases was the robot able to correctly map the environment. In contrast, our approach using the additional constraint always generated an accurate map. One example is shown in Figure 11. Here, the robot stopped the loop-closing after traversing half of the inner loop. In both cases we used 80 particles.

As this experiment illustrates, the termination of the loop-closing is important for the convergence of the filter and to obtain accurate maps in environments with several (nested) loops. Note that similar results in principle can also be obtained without this termination constraint if the number of particles is dramatically increased. Since exploration is an online problem and each particle carries its own map it is of utmost importance to keep the number of particles as small as possible. Therefore, our approach can also be regarded as a contribution to limit the number of particles during FastSLAM.

\subsection{Evolution of $N_{\text {eff }}$}

In this experiment, we demonstrate the behavior of the optional termination criterion that triggers the active loop-closing behavior. Additionally to the constraint that the uncertainty $\mathcal{H}(t)$ must be bigger than the uncertainty at the entry point $\mathcal{H}\left(t_{e}\right)$ of the loop, the process is stopped whenever the effective number of particles $N_{\text {eff }}$ stays constant for a certain period of time. This criterion, which can be applied if the underlying mapping system uses an adaptive resampling technique [9], was introduced to avoid that the robot moves through the loop even if no new information can be obtained from the sensor data. The robot re-traverses the loop only as long as the sensor data is useful to identify unlikely hypotheses about maps and poses.

One typical evolution of $N_{\text {eff }}$ is depicted in the left image of Figure 12. To achieve a good visualization of the evolution of $N_{\text {eff }}$, we processed a recorded data file using 150 particles. Due to the adaptive resampling strategy, only very few resampling operations were needed. The robot started at position A 

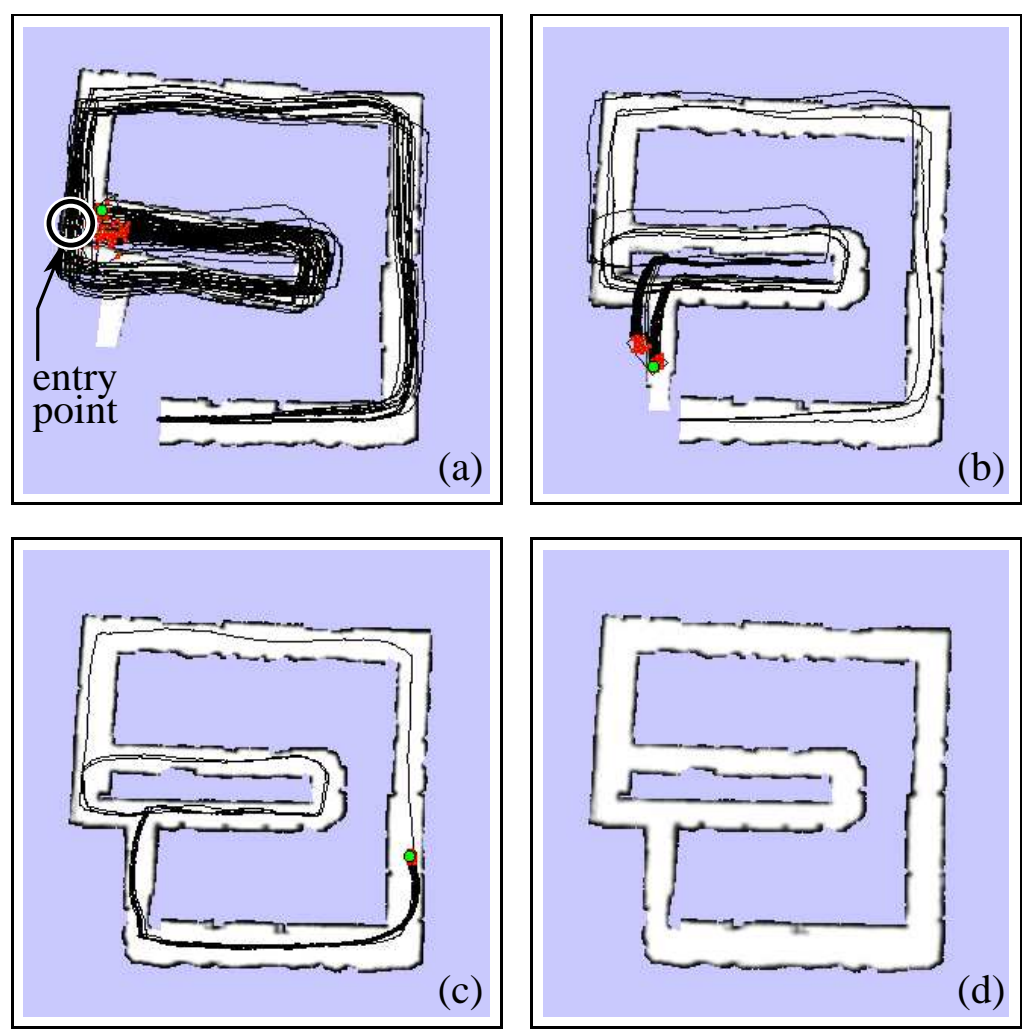

Figure 11: In image (a), the robot detected an opportunity to close a loop. It traversed parts of the inner loop as long as its uncertainty exceed the uncertainty $\mathcal{H}\left(t_{e}\right)$ of the posterior when the robot at the entry point and started the loop-closing process. The robot then turned back and left the loop (b) so that enough hypotheses survived to correctly close the outer loop (c) and (d). In contrast, a system considering only a constant threshold criterion fails to map the environment correctly as depicted in Figure 6 .
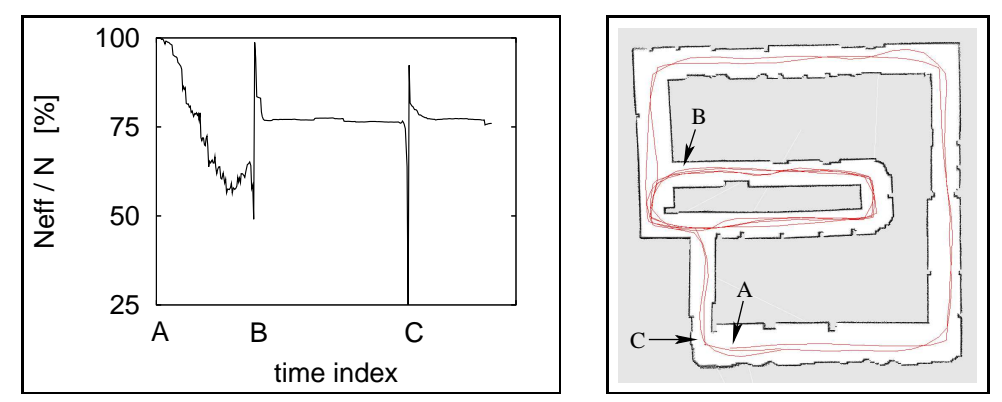

Figure 12: The graph plots the evolution of the $N_{\text {eff }}$ function over time during an experiment carried out in the environment shown in the right image. The robot started at position A. The position B corresponds to the closure of the inner loop, and C corresponds to closure of the outer loop. 
and in the first part of the experiment moved through unknown terrain (between the positions $\mathrm{A}$ and B). As can be seen, $N_{\text {eff }}$ decreases over time. After the loop has been closed correctly and unlikely hypotheses had partly been removed by the resampling action (position B), the robot re-traversed the inner loop and $N_{\text {eff }}$ stayed more or less constant. This indicates that acquiring further data in this area has only a small effect on the relative likelihood of the particles and the system could not determine which hypotheses represented unlikely configurations. In such a situation, it therefore makes more sense to focus on new terrain acquisition and to not continue the loop-closing process.

Furthermore, we analyzed the length of the trajectory traveled by the robot. Due to the active loopclosing, our technique generates longer trajectories compared to a purely frontier-based exploration strategy. We performed several experiments in different environments in which the robot had the opportunity to close loops and measured the average overhead. During our experiments, we observed an overhead varying from $3 \%$ to $10 \%$, but it obviously depends on number of loops in the environment.

\subsection{Multiple Nested Loops}

To illustrate, that our approach is able to deal with several nested loops, we performed a simulated experiment shown in Figure 13. The individual images in this figure depict eight snapshots recorded during exploration. Image (a) depicts the robot while exploring new terrain and image (b) while actively closing the most inner loop. After that, the robot focused on acquiring so far unknown terrain and maps the most outer loop as shown in (c) and (d). Then the robot detects a possibility to close a loop (e) and follows its previously taken trajectory (f). After aborting the loop closing behavior, the robot again explores the loop in the middle (g), again closes the loop accurately, and finishes the exploration task (h).

\subsection{Computational Resources}

Note that our loop-closing approach needs only a few additional resources. To detect loops, we maintain an additional topological map for each particle. These topological maps are stored as a graph structure and for typical environments only a few kilobytes of extra memory is needed. To determine the distances based on the grid map in Eq. (3) and (4), our approach directly re-uses the result of a value iteration (alternatively Dijkstra's algorithm) computed on the most likely grid map, which has already been computed in order to evaluate the frontier cells. Only the distance computation using the topological map needs to be done from scratch. However, since the number of nodes in the topological map is much smaller than the number of grid cells, the computational overhead is comparably small. In our experiments, the time to perform all computations in order to decide where to move next increased by around $10 \mathrm{~ms}$ on a standard $\mathrm{PC}$ when activation the active loop-closing technique. 


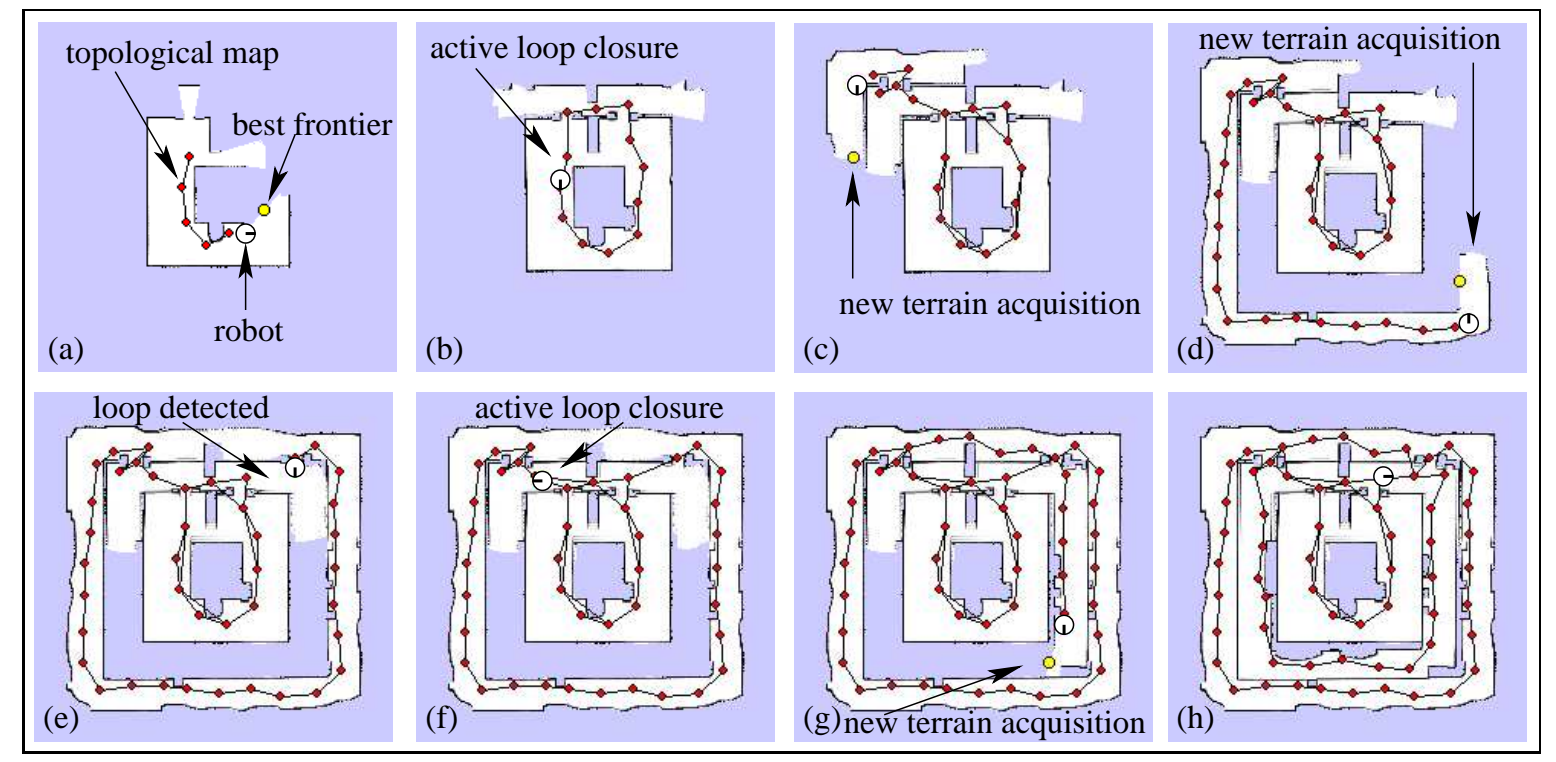

Figure 13: Snapshots during the exploration of a simulated environment with several nested loops. The red circles represent nodes of the topological map plotted on top of the most likely grid map. The yellow circle corresponds to the frontier cell the robot currently seeks to reach.

\section{Conclusions}

In this paper, we presented a novel approach for active loop-closing during autonomous exploration. We combined a Rao-Blackwellized particle filter for localization and mapping with a frontier-based exploration technique extended by the ability to actively close loops. Our algorithm forces the robot to re-traverse previously visited loops and in this way reduces the uncertainty in the pose estimate. As a result, we obtain more accurate maps compared to standard combinations of SLAM algorithms with exploration techniques. As fewer particles need to be maintained to build accurate maps, our approach can also be regarded as a contribution to limit the number of particles needed during FastSLAM.

\section{Acknowledgment}

This work has partly been supported by the German Science Foundation (DFG) under contract number SFB/TR-8 (project A3) and by the EC under contract number FP6-004250-CoSy.

\section{REFERENCES}

[1] F. Bourgoult, A.A. Makarenko, S.B. Williams, B. Grocholsky, and F. Durrant-Whyte. Information based adaptive robotic exploration. In Proc. of the IEEE/RSJ Int. Conf. on Intelligent Robots and Systems (IROS), Lausanne, Switzerland, 2002. 
[2] W. Burgard, M. Moors, C. Stachniss, and F. Schneider. Coordinated multi-robot exploration. IEEE Transactions on Robotics, 2005. To appear.

[3] F. Dellaert, D. Fox, W. Burgard, and S. Thrun. Monte carlo localization for mobile robots. In Proc. of the IEEE Int. Conf. on Robotics \&3 Automation (ICRA), Leuven, Belgium, 1998.

[4] G. Dissanayake, H. Durrant-Whyte, and T. Bailey. A computationally efficient solution to the simultaneous localisation and map building (SLAM) problem. In ICRA'2000 Workshop on Mobile Robot Navigation and Mapping, San Francisco, CA, USA, 2000.

[5] A. Doucet, J.F.G. de Freitas, K. Murphy, and S. Russel. Rao-blackwellized partcile filtering for dynamic bayesian networks. In Proc. of the Conf. on Uncertainty in Artificial Intelligence (UAI), Stanford, CA, USA, 2000.

[6] A. Eliazar and R. Parr. DP-SLAM: Fast, robust simultainous localization and mapping without predetermined landmarks. In Proc. of the Int. Conf. on Artificial Intelligence (IJCAI), Acapulco, Mexico, 2003.

[7] H. Feder, J. Leonard, and C. Smith. Adaptive mobile robot navigation and mapping. Int. Journal of Robotics Research, 18(7), 1999.

[8] R. Grabowski, P. Khosla, and H. Choset. Autonomous exploration via regions of interest. In Proc. of the IEEE/RSJ Int. Conf. on Intelligent Robots and Systems (IROS), Las Vegas, NV, USA, 2003.

[9] G. Grisetti, C. Stachniss, and W. Burgard. Improving grid-based slam with rao-blackwellized particle filters by adaptive proposals and selective resampling. In Proc. of the IEEE Int. Conf. on Robotics $\mathcal{E}$ Automation (ICRA), pages 2443-2448, Barcelona, Spain, 2005.

[10] J.-S. Gutmann and K. Konolige. Incremental mapping of large cyclic environments. In Proc. of the IEEE Int. Symposium on Computational Intelligence in Robotics and Automation (CIRA), pages 318-325, Monterey, CA, USA, 1999.

[11] D. Hähnel, W. Burgard, D. Fox, and S. Thrun. An efficient FastSLAM algorithm for generating maps of large-scale cyclic environments from raw laser range measurements. In Proc. of the IEEE/RSJ Int. Conf. on Intelligent Robots and Systems (IROS), Las Vegas, NV, USA, 2003.

[12] S. Koenig and C. Tovey. Improved analysis of greedy mapping. In Proc. of the IEEE/RSJ Int. Conf. on Intelligent Robots and Systems (IROS), Las Vegas, NV, USA, 2003.

[13] J.S. Liu. Metropolized independent sampling with comparisons to rejection sampling and importance sampling. Statist. Comput., 6:113-119, 1996.

[14] A.A. Makarenko, S.B. Williams, F. Bourgoult, and F. Durrant-Whyte. An experiment in integrated exploration. In Proc. of the IEEE/RSJ Int. Conf. on Intelligent Robots and Systems (IROS), Lausanne, Switzerland, 2002. 
[15] M. Montemerlo, S. Thrun, D. Koller, and B. Wegbreit. FastSLAM: A factored solution to simultaneous localization and mapping. In Proc. of the National Conference on Artificial Intelligence (AAAI), Edmonton, Canada, 2002.

[16] H.P. Moravec and A.E. Elfes. High resolution maps from wide angle sonar. In Proc. of the IEEE Int. Conf. on Robotics \& Automation (ICRA), pages 116-121, St. Louis, MO, USA, 1985.

[17] K. Murphy. Bayesian map learning in dynamic environments. In Neural Info. Proc. Systems (NIPS), Denver, CO, USA, 1999.

[18] N. Roy, M. Montemerlo, and S. Thrun. Perspectives on standardization in mobile robot programming. In Proc. of the IEEE/RSJ Int. Conf. on Intelligent Robots and Systems (IROS), Las Vegas, NV, USA, 2003.

[19] R. Sim, G. Dudek, and N. Roy. Online control policy optimization for minimizing map uncertainty during exploration. In Proc. of the IEEE Int. Conf. on Robotics \& Automation (ICRA), New Orleans, LA, USA, 2004.

[20] C. Stachniss and W. Burgard. Exploring unknown environments with mobile robots using coverage maps. In Proc. of the Int. Conf. on Artificial Intelligence (IJCAI), pages 1127-1132, Acapulco, Mexico, 2003.

[21] S. Thrun. An online mapping algorithm for teams of mobile robots. Int. Journal of Robotics Research, 2001.

[22] R. van der Merwe, N. de Freitas, A. Doucet, and E. Wan. The unscented particle filter. Technical Report CUED/F-INFENG/TR380, Cambridge University Engineering Department, August 2000.

[23] G. Weiß, C. Wetzler, and E. von Puttkamer. Keeping track of position and orientation of moving indoor systems by correlation of range-finder scans. In Proc. of the IEEE/RSJ Int. Conf. on Intelligent Robots and Systems (IROS), pages 595-601, Munich, Germany, 1994.

[24] B. Yamauchi. Frontier-based exploration using multiple robots. In Proc. of the Second International Conference on Autonomous Agents, pages 47-53, Minneapolis, MN, USA, 1998. 